\title{
Supporting the reuse of effective CSCL learning designs through social structure representations
}

\author{
Authors: Serena Alvino a; Juan I. Asensio-Pérez ${ }^{\text {b; }}$ Yannis Dimitriadis ${ }^{\text {b }}$; Davinia \\ Hernández-Leo ${ }^{\mathrm{c}}$ \\ Affiliations: ${ }^{a}$ University of Genoa and Institute for Educational Technologies, Italy \\ b School of Telecommunications Engineering, University of Valladolid, \\ Spain \\ c Department of Technologies, Pompeu Fabra University, Barcelona, \\ Spain
}

DOI: $10.1080 / 01587910903023215$

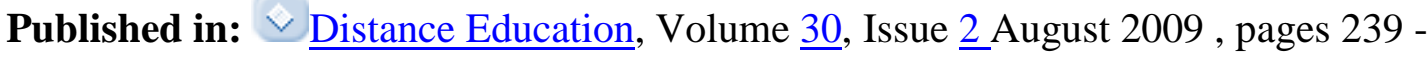
258

\section{Abstract}

Distance and blended collaborative learning settings are usually characterized by different social structures defined in terms of groups' number, dimension, and composition; these structures are variable and can change within the same activity. This variability poses additional complexity to instructional designers, when they are trying to develop successful experiences from existing designs. This complexity is greatly associated with the fact that learning designs do not render explicit how social structures influenced the decisions of the original designer, and thus whether the social structures of the new setting could preclude the effectiveness of the reused design. This article proposes the usage of new representations (social structure representations, SSRs) able to support unskilled designers in reusing existing learning designs, through the explicit characterization of the social structures and constraints embedded either by the original designers or the reusing teachers, according to well-known principles of good collaborative learning practice. The article also describes an evaluation process that involved university professors, as well as the main findings derived from it. This process supported the initial assumptions about the effectiveness of SSRs, with significant evidence from both qualitative and qualitative data.

Keywords: learning design; computer-supported collaborative learning; collaborative scripts; social structures

\section{Introduction}

Recent approaches to instructional design (ID) point out that the design process, as put into practice by expert designers, is not a procedure but a problem-solving process. It can be assumed that it is not possible to identify a perfect solution, since reality is often ill-structured and rich in complex variables. Heuristics and good practices held as effective for a specific problematic situation guide skilled designers through different decision-making steps, which progressively contextualize the design by introducing constraints (Greeno, Korpi, Jackson, \& Michalchik, 1990; Silber, 2007). The key role 
played by ID heuristics and practices in this view makes the prospect of rendering explicit, formalizing, sharing, and reusing them in different contexts more and more important in order to support unskilled designers in the decision-making steps involved in traditional, as well as in open, distance, and flexible learning design processes. This prospect has become especially significant for the field of computer-supported collaborative learning (CSCL) in which computer-mediated (face-to-face or distance) interactions among learners are promoted as a key factor for improving learning, but where good practices about how to structure these interactions have until now hardly been shared by experts. Recently, the bridge between collaborative learning and traditional ID methods (Dillenbourg, 2002) by means of CSCL scripts has attracted much attention. CSCL scripts computationally specify, sequence, and distribute the roles and activities involved in a CSCL situation, thus providing a certain degree of scaffolding for fostering learners' engagement in more potentially effective interactions (Weinberger, Collar, Dimitriadis, Mäkitalo-Siegl, \& Fischer, 2008).

The inherent complexity of collaboration structures and the need to avoid excessive coercion in scripted collaboration (Dillenbourg, 2002), among other factors, make the process of creating effective collaborative learning designs in the form of CSCL scripts a daunting task for unskilled instructional designers or practitioners. Several types of research initiatives have tackled this challenge by investigating the creation of communities in which CSCL scripts are shared and exchanged (see, for instance, the Cloudworks initiative at the Open University in the UK, at http://cloudworks.open.ac.uk/); defining CSCL script design processes based on the reuse of patterns, exemplars, and other script components (Hernández-Leo, Harrer, Dodero, Asensio-Pérez, \& Burgos, 2007); and developing CSCL script authoring tools based on practitioner-friendly abstractions and graphical representations (HernándezLeo, Villasclaras-Fernández, Asensio-Pérez, \& Dimitriadis, 2007). The intense international research effort is also exemplified by several workshops at international conferences (e.g., Vignollet, David, Ferraris, Martel, \& Lejeune, 2006); research projects and European research teams within the Kaleidoscope Network of Excellence (Kaleidoscope, 2003-2009); specific international books (Botturi \& Stubbs, 2007), and standardization processes, for example, within the IMS Global Learning Consortium (IMS Global Learning Consortium, 2003).

This article focuses on a particular design problem that unskilled instructional designers or practitioners encounter when trying to create-by-reuse an existing CSCL script in their particular learning context, the so-called script instantiation problem. This problem is one significant example of the importance of rendering explicit the constraints that the original designer considered when making design decisions. The lack of information about those constraints could lead an unskilled designer to develop an ineffective CSCL script. To address the problem, the article proposes, illustrates, and provides evidence in support of a new formalism for modelling and visualizing social structures involved in a CSCL activity, called social structure representations (SSRs). SSRs are intended to complement existing design processes for CSCL scripts, so that unskilled designers may be aware of and consider important constraints when designing social structures (e.g., number of groups, dimension, composition) for their particular learning environment (including resources, tools, and services). Note that the issue addressed in this article is particularly significant in distance learning situations in which interactions are necessarily mediated by information and communication technology tools, since the effectiveness of the design decisions may be valid only for a 
given set of tools. Further, flexibility is even more necessary in the case of complex collaborative learning situations, such as the ones studied in this article.

The article is structured as follows. Section 2 introduces and defines the instantiation problem tackled by the article and illustrates it by means of a case study. Section 3 introduces the SSR proposal, and Section 4 describes the SSR evaluation process which was undertaken. That evaluation process, based on a mixed quantitative-qualitative evaluation method, engaged real practitioners (university teachers) who were unskilled in CSCL in a process of reusing an existing CSCL script for realistic design scenarios which were formalized with the IMS Learning Design (IMS LD) educational modelling language (IMS Global Learning Consortium, 2003). These educators compared the complexity and effectiveness of reusing the script with or without the help of SSRs. Finally, Section 5 concludes the article and outlines some future short- and mediumterm research lines derived from the findings.

\section{The instantiation problem when reusing CSCL scripts}

Usually a learning design process starts from a general idea of an effective learning path and develops through different steps by contextualizing the design until the implementation of a session (Silber, 2007). In particular, the design of CSCL scripts is a process, spanning from their conception to their execution, which comprises different phases in which the designers progressively enrich the script by elaborating a range of aspects, such as structure, activity description, content-specific resources, supporting computing tools, and group structure. Several CSCL script design processes can be found in the literature (e.g., Dillenbourg \& Tchounikine, 2007; Hernández-Leo et al., 2006; Weinberger et al., 2008), each one proposing a different set of phases that should lead to a ready-to-execute script. Script authoring and script instantiation are commonly considered to be two important phases in existing CSCL script design processes.

Although there is no consensus on the number, nature, sequence, or even the terminology of the involved phases, authoring comprises the steps performed by the designer to create a potentially effective general script, while instantiation comes later and includes the specific characteristics and data for a script to be run in a specific learning situation. This article focuses on formalized CSCL learning designs which have been authored but not yet instantiated, in particular on the problems that arise when trying to reuse a CSCL script that has been created for a specific setting (and maybe instantiated in it) in a different educational context.

Section 2.1 firstly presents a pattern-based CSCL script authoring process proposed by the authors and described in Hernández-Leo et al. (2006). This process, which has been implemented in an authoring tool, supports unskilled designers and practitioners in the creation of CSCL scripts formalized with IMS LD (IMS Global Learning Consortium, 2003). Secondly, it outlines a script instantiation process, showing how it is normally separate from the authoring phase to facilitate the reuse of the script in different educational contexts. Starting from these premises, Section 2.2 shows how this division can generate problems when a third-party teacher reuses the script and re-contextualizes it in a new educational context. 


\subsection{Authoring and instantiating a collaborative script}

The CSCL script design process proposed by Hernández-Leo, Asensio-Pérez, and Dimitriadis (2005) is based on the selection and combination of so-called collaborative learning flow patterns (CLFPs). The good practices for collaborative learning contained in CLFPs are formalized with the IMS LD specification language to create a CSCL script which can be instantiated into specific learning scenarios. An IMS LD authoring tool specialized for CSCL called Collage (Hernández-Leo et al., 2006) supports this CLFP-based script design process (see also http://ulises.tel.uva.es/collage). The usage of patterns is a key element of this process as it enables existing collaborative learning techniques that have proven to be effective to be reused by unskilled instructional designers or practitioners. Examples of collaborative learning techniques formalized as CLFPs include Brainstorming and Jigsaw. For a complete description of Collage CLFPs, please visit http://ulises.tel.uva.es/collage/clfps.

One of the cases in which the CLFP-based script design process has been applied was in a blended course on grid computing technologies that took place in March 2007 within the context of a Spanish R\&D project called MOSAIC. The MOSAIC course involved $12 \mathrm{PhD}$ students from three universities (with four students from each site). Their final goal was to collaboratively create a concept map on the topic of grid computing after reading a set of basic scientific papers about the subject. Four synchronous sessions lasting a total of 8.5 hours were available for performing a set of learning activities aimed at achieving that final goal. The technical support for the course was provided by the .LRN (‥d.) learning management system (LMS), which includes an IMS LD interpreter (Escobedo, de la Fuente, Gutiérrez, Pardo, \& Delgado, 2007).

The script authoring phase of the CLFP-based design process comprises two main steps supported by the Collage tool:

- Firstly, the Collage user (teacher or instructional designer) is provided with a detailed description of the CLFPs in terms of the types of learning objectives and tasks they are suitable for. Using that information, the Collage user selects one or several CLFPs. (CLFPs can be combined by structuring one activity of a CLFP according to the sequence of activities prescribed by another one.) For the MOSAIC situation, three CLFPs (Pyramid, Jigsaw, Think-Pair-Share) were selected and combined (as shown in Figure 1). For instance, the Jigsaw CLFP was selected to foster interactions potentially effective for promoting positive interdependence, discussion, and individual accountability.

- Secondly, the Collage user customizes the selected CLFPs by including the description of activities and roles, as well as the resources (learning objects and computing tools) to be used in each activity. 




[Enlarge Image]

Figure 1. CSCL script of the MOSAIC course, as represented in the Collage tool.

For the MOSAIC course, for example, following the Jigsaw prescriptions, students were divided into discussion groups and assigned three papers about a specific topic intended to increase their expertise in the topic. After the discussion in the expert groups, the groups were reorganized into new Jigsaw groups. In the Jigsaw group, students had to explain their ideas on their expert subjects to others and then had to collaborate to propose a joint conceptual map. The script designers also gave specific references about the tools that should be provided to support each activity.

The final product of the above two steps is a so-called IMS LD Unit of Learning (UoL) that contains a structured XML document with all the information provided by the Collage user, as well as the learning material (e.g., electronic documents) and the tools (or references to them) that are intended to be used throughout the script enactment with the real users.

After the described script authoring phase, and before its enactment, a UoL must undergo the so-called production phase (IMS Global Learning Consortium, 2003). During production a UoL is instantiated by specifying the number of groups, the involvement of participants in the different learning activities, possible moderators or experts, and the specific schedule. As we mentioned before, the division between the authoring phase of a UoL and its instantiation is intended to facilitate the reuse of the UoL in different educational contexts. 
In the MOSAIC course, the course designers created four Jigsaw groups (each with three participants) whose members were expected to carry out some of their activities in four expert groups (of four participants each).

However, the instantiation phase has not yet been addressed in a standard way by the community. In the MOSAIC case, the UoL instantiation was carried out by a specific tool provided by the LMS, while an instantiation tool specifically applicable to UoLs created by Collage is now available. That tool, called InstanceCollage (HernándezGonzalo, Villasclaras-Fernández, Hernández-Leo, Asensio-Pérez, \& Dimitriadis, 2008), uses the same graphical abstractions characterizing Collage and takes into account some inherent restrictions of the CLFPs when creating and populating groups, thus facilitating the instantiation process in complex collaborative learning scenarios.

\subsection{Issues in reusing a collaborative script}

Taking into account the description of the MOSAIC course we can now go on to analyse the issues that may arise when a new, unskilled designer wants to reuse such a design and instantiate it for execution in different environments.

The designers of MOSAIC used Collage to create a UoL to be run in a specific predefined context, and in the authoring phase took into account several constraints for runtime, concerning the instantiation phase. For example, they considered the number and characteristics of students attending the course (12 PhD students from three Spanish universities) and the available time (two weeks), as well as the specific aim of the group activity (collaborative construction of a shared conceptual map). On the basis of these contextual constraints, they identified their communication needs and the necessary tools. Then, in the script instantiation process they created small collaborative groups which would work at distance to produce an artefact and provided these groups with specific editing tools and a synchronous communication tool. As it can be inferred by these premises, in the MOSAIC context this UoL turned out to be very effective when it was put in practice with the real participants of the course (de la Fuente, Pardo, Delgado, Asensio, \& Dimitriadis, 2008); but what would happen if other teachers decide to reuse this UoL in their own educational context?

IMS LD has not addressed satisfactorily the complexity of collaborative activities, and therefore several problems have been reported in modelling collaborative learning processes, which are related, among other things, to defining groups or structuring the flow of collaborative learning activities (Hernández-Leo et al., 2005; Miao, Hoeksema, Hoppe, \& Harrer, 2005). Specific tools such as Collage or Recourse (see

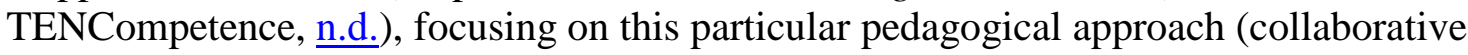
learning), present to their users only the required functionality (Hernández-Leo et al., 2005), and so overcome the complexity of authoring a collaborative script (Koper, 2006). Unfortunately, teachers reusing a collaborative UoL (independently of the authoring tool used by the creator of the learning design), have to face other problems concerning the instantiation process.

The MOSAIC UoL contains well-defined objectives, prerequisites, content, didactic strategies, activities, and task descriptions, as well as suggestions for implementation. It 
also embeds a selection of resources and links to the tools required by each phase of the activity. However, the flow-centred representation of the learning process, which characterizes IMS LD specification, lacks important information about, for example, flows of resources and artefacts, the need for reference experts or moderators, and especially the structure of the learning groups, in terms of their number, dimension, and composition (Persico \& Sarti, 2005). This is partially due to the fact that in IMS LDcompliant authoring tools the learning design is taken as a starting point to solve educational problems (Koper, 2006). Nevertheless, when using an instantiation tool, such as InstanceCollage, to generate a specific session, a teacher has to make some key design decisions concerning just these important aspects.

In addition, a UoL normally introduces into the design process a range of constraints which are naturally embedded in the activities defined by the designer. In fact, as mentioned before, a design process usually occurs by a progressive contextualization because each decision or choice progressively contextualizes the design, introducing constraints (Silber, 2007). When reusing a script a teacher integrates in his/her own design process the product of another design process at a particular contextualization level. Thus, he/she should be well aware of the constraints characterizing both his/her own context and the script (Alvino, 2008). In a CSCL design process we can identify three main types of constraints (Alvino, 2008): (1) initial - technical, financial, and contextual constraints characterizing the specific learning context; (2) structural constraints deriving from choices and decisions made during the design process which affect other choices (i.e., objectives, contents); and (3) heuristic - constraints related to the application of heuristics and good practices for effectively structuring the community of learning, organizing collaborative activities, and modelling the learning environment.

Reusable scripts are normally characterized by structural and heuristic constraints. For example, the need for a decomposable topic is structurally embedded in the Jigsaw pattern, since it is based on discussion in expert groups, which is then followed by work in the heterogeneous Jigsaw groups to solve a problem. To be effective, the activity requires as many expert groups as the number of subtopics, or a multiple of it. This information can be made explicit in a UoL only in an open-text field by a very scrupulous designer. Otherwise, designers reusing a Jigsaw UoL would face an important hidden structural constraint. Alternatively, for example, heuristic constraints can be identified in a UoL in relation to the use of particular communication tools or with the aim of groups (e.g., production of an artefact or decision-making) (Zang \& Ge, 2005). In fact, according to literature on design good practices (Kabanoff \& O'Brien, 1979; Trentin, 2001; Zang \& Ge, 2005), the definition of the aim affects choices about the number of groups (e.g., four Jigsaw groups), their dimensions (e.g., three persons in a Jigsaw group) and compositions (in terms of roles involved, required competencies, or need of homogeneity or heterogeneity), the need for guidance and particular communication tools (synchronous or asynchronous).

The aforementioned information is not rendered explicit in an IMS LD UoL because this is not information required by the specification. Thus, for example, an unskilled teacher reusing the UoL instantiated in the MOSAIC course might create a session characterized by groups of 15 students who should interact in a chat to produce an artefact, without any guidance of a moderator. A skilled practitioner will realize that these design choices are unlikely to result in an effective learning experience because, 
for example, the effectiveness of a communication tool in enabling a predefined objective is strictly connected with the number of users. In addition, a group aimed at producing an artefact should be composed of a small number of members because decision-making and ongoing-mediation processes are difficult to carry out in large groups (Kabanoff \& O'Brien, 1979; Trentin, 2001).

In conclusion, we argue that when reusing a collaborative UoL, teachers need important information about how to structure an effective session and need to be aware of all the constraints (structural or heuristic) inherent in the script. However, current learning designs cannot effectively support such a process because the information required is not made explicit. In the following sections, the SSR is proposed as a solution to this problem and the findings from an evaluation of the approach by practitioners, employing the MOSAIC situation, is presented.

\section{Enhancing CSCL scripts reuse by sharing design practice representations: the SSRs}

As mentioned before, a collaborative UoL is more focused on the activity flow than on its structures, especially concerning groups. While resources and services associated with each phase of the activity are made explicit, the social structures of groups that follow one another in the activity flow are usually not described. According to Persico and Sarti (2005), social structures denote group configurations in terms of number, dimension, composition, and participants' tasks. Only this last characteristic is expressed in IMS LD in terms of roles.

Persico and Sarti (2005) argued that generally instructional designers refer to heuristics or best practices in order to form groups, making decisions about size, heterogeneity, and participants' tasks, taking into account contextual constraints such as the number of course participants, tutors available, the type of activity to be carried out, and the participants' competence; in most cases decisions regarding social structures impact on the affordances that the communication environment has to offer and favour the interaction flow in the desired directions.

These heuristics can be rendered explicit to support unskilled teachers in the design of open, distance, and flexible learning processes (Alvino, 2008). In particular, these formalized practices can be very useful to foster an effective reuse of CSCL scripts, which are often characterized by different structural and heuristic constraints concerning collaborative groups' social structures (Alvino, 2008). Formalisms such as UML diagrams, conceptual maps, flowcharts, and decision trees are widely used in education to represent and clarify complex relationships or flows (Paquette, Léonard, LudgrenCayrol, Mihaila, \& Gareau, 2005) and to render explicit, share, and reuse their heuristics. However, teachers need an appropriate means which makes apparent the critical features of their design, such as groups' social structures and the relative constraints. Starting from these premises, we propose the SSRs as an innovative means which allows for modelling and visualizing social structures involved in CSCL activities. 
Information about how to instantiate effective social structures can be very important to support an unskilled teacher in reusing a UoL. In addition, many structural and heuristic constraints characterizing the script affect group structuring. Starting from these premises, we tried to integrate the use of IMS LD in the design process to specify aspects of the design with other representations that allow for modelling and visualization of social structures involved in a CSCL activity using SSRs.

SSRs propose a graphical way to represent the solution to an educational problem in a CSCL context, through a set of schemata. The problem can be formalized at different levels of abstraction; that is, SSRs can be associated both with general, abstract scripts (such as a pedagogical pattern) and with more contextualized and formalized ones (such as UoLs). In particular, they focus on a particular task or activity and formalize a picture of the social structures characterizing groups (in terms of their number, their dimension, and their composition), also illustrating their relations to the learning environment. Each schema describes a particular phase of the activity according to a unifying template composed of three main sections:

- Resources: The SSR represents the resources of the activity (for example, reference materials, groups' artefacts, bibliographies, Web references) as an input or as an output of the process (signified by the direction of the arrows). It also relates each resource to individual students, roles, or groups.

- Community of learning: The SSR represents groups in terms of their social structures, including their number, dimension, and composition. In addition, experts or moderators who could be useful in the activity are represented as playing a mediatory function between students and resources or tools.

- Events, services, or tools: The SSR is subdivided into two main sections, devoted to representing events, services, and tools that support distant or face-toface sessions. When SSRs are formalized at an abstract level this information is represented in terms of the functions which events, services, or tools can play in a CSCL environment (Koschmann, 1996).

SSRs support the representation of an educational problem by focusing on social structures, and they may be associated with a learning design in order to complement the information embedded in it. In particular, they may overcome some limitations of IMS LD UoLs by providing an additional, complementary representation of collaborative activities and the groups they involve.

SSRs are not supposed to be self-contained but they should be associated with a learning design in order to complement the information embedded in it. The educational problem can be formalized at different levels of abstraction; that is, SSRs can be associated both with general, abstract scripts (such as a pedagogical pattern) and with more contextualized and formalized ones (such as UoLs). In particular, these representations may overcome some limitations of scripts based on IMS LD or other languages by providing an additional, complementary representation of collaborative activities and the groups they involve.

Similarly to this proposal based on the use of SSRs, there are other research initiatives (Botturi \& Stubbs, 2007; Paquette et al., 2005; Persico \& Sarti, 2005) dealing with visual artefacts for supporting educational modelling and ID tasks. Some of those initiatives tackle the problems of instantiating CSCL scripts. However, none of them 
has studied the particular issue of modelling design decisions with respect to social structuring in order to improve reusability of existing scripts.

SSRs can also support unskilled teachers in the instantiation of a learning design, such as a UoL, because they represent all the structural and heuristic constraints inherent in the learning design itself. While structural constraints are formalized as static, independent variables for a specific phase or task, the heuristic ones are expressed in terms of ranges of possibilities. Figure $\underline{2}$ shows an SSR representing the 'expert group' phase of the Jigsaw activity as formalized in the UoL implemented in the MOSAIC course. In the upper section resources involved in the 'expert group' phase are represented, including reference papers and individual and collaborative artefacts (which are considered both as outputs and as inputs to the process). In the middle section the community of learning is illustrated by homogeneous groups and moderators, with geometric figures representing individuals characterized by similar properties which are relevant for the specific task (in this case, students who read the same papers). In the lower section we show that this is a distance phase of the activity supported by a repository, a concept mapping tool, editing tools, and an instant messaging tool.

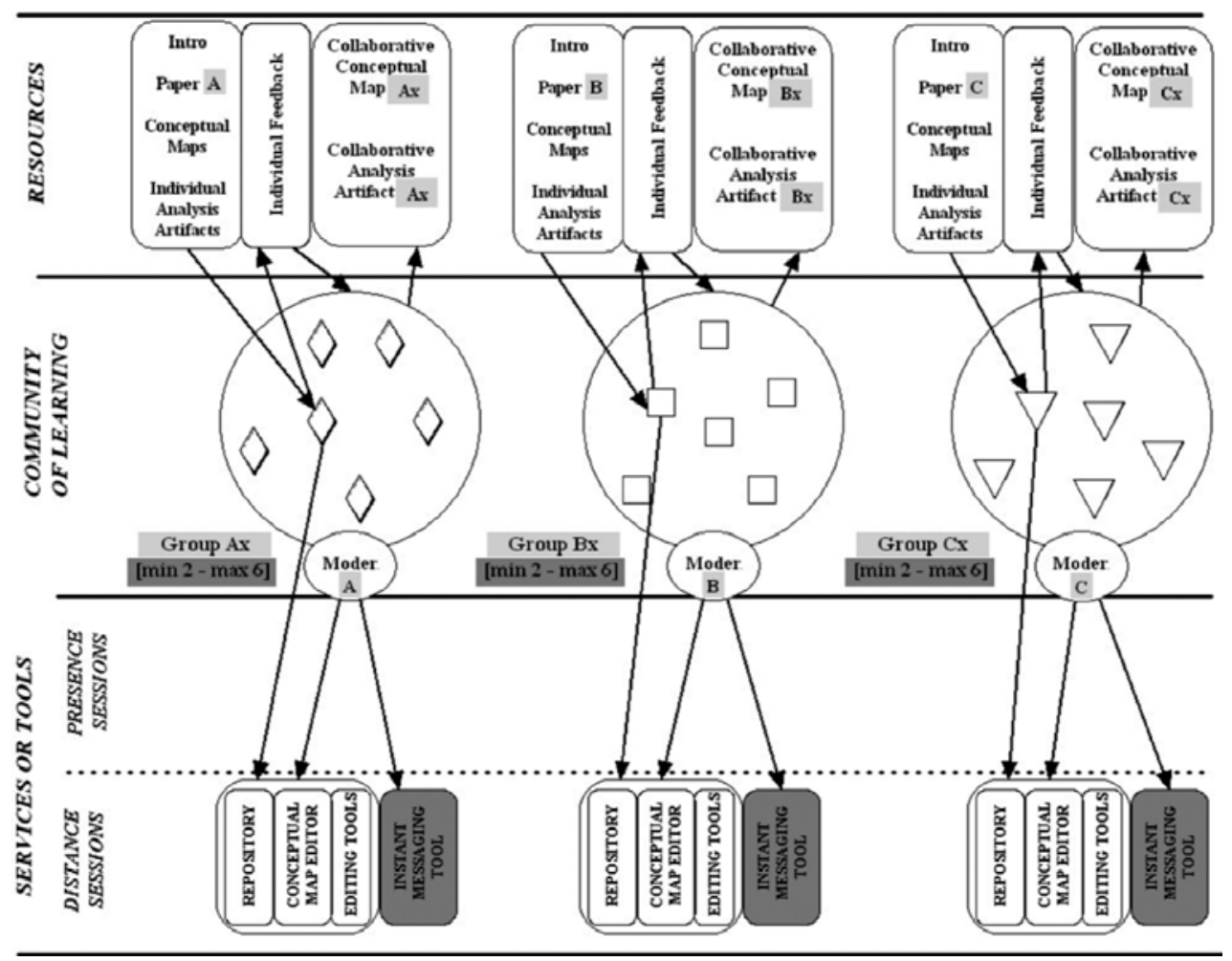

[Enlarge Image]

Figure 2. Instance level SSR of the MOSAIC Jigsaw's expert groups phase.

The schema in Figure $\underline{2}$ also represents two main constraints characterizing the specific expert group phase. By means of different colours (seen here in grey), it represents both fixed design elements and dependent variables (in terms of ranges of possibilities). We may also observe a structural constraint (see Section 2.2) in the first two sections: the need for a decomposable topic embedded in the Jigsaw activity (subtopics A, B, C) and the necessity for as many 'expert groups' as the number of subtopics or a multiple of it (groups $A x, B x, C x$, where $x 1$ ). In the second and in the third sections, a heuristic 
constraint is shown concerning the dimension of expert groups. Since the groups are to collaboratively produce artefacts by communicating through an instant messaging tool, according to principles of good practice in CSCL (Kabanoff \& O'Brien, 1979; Trentin, 2001; Zang \& Ge, 2005), groups should be composed of a maximum of six members.

Thus, one may expect that SSRs provide teachers with important complementary information about scripts that is not usually explicit in learning designs, such as those that refer to social structures and constraints. In the following section, an evaluation process that aims to analyse the support SSRs provide to unskilled practitioners is described.

\section{Evaluation of SSRs}

In this article we argue that, because of their support for formulating shared representations of group social structures, SSRs play a complementary role to learning designs (UoLs) and support unskilled teachers in reusing and instantiating scripts for their particular educational context.

To test this idea we undertook an evaluation process (Stake, 1995) focusing on three main issues of interest in the design process:

1. when reusing UoLs, unskilled teachers lack information about how to effectively instantiate them, especially in terms of groups structuring;

2. SSRs provide complementary information to the ones provided by learning designs (UoLs);

3. SSRs effectively support unskilled teachers in reusing and instantiating scripts.

These issues have been investigated by actively involving unskilled teachers in a design process focused on the analysis of a predefined UoL and on its instantiation into different scenarios. Although the design process is controlled, the predefined and new scenarios are strongly based on realistic contexts (in this case, the MOSAIC course). Qualitative and quantitative methods were used to collect data about teachers' choices and opinions in two different design situations, with and without the support of SSRs. In the following subsections, the methodological aspects are described first, followed by a discussion of the results.

\subsection{The methodology of the evaluation process}

The evaluation process involved 10 Italian university professors, who were selected on the basis of similar characteristics in terms of educational skills and experience with online learning design. For different reasons, all of the teachers were interested in online learning and thus they were eager to participate in the proposed controlled experience. Some of them had just begun to manage their own online academic courses, while others had already been working in various online tutoring activities, although none of them had specific online learning design skills. 
The teachers were engaged in a highly structured design process including five main phases (see Figure $\underline{3}$ ).

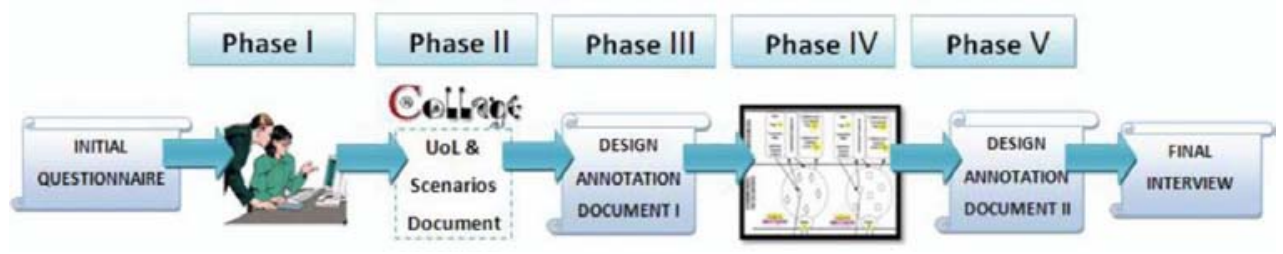

[Enlarge Image]

Figure 3. Overview of the evaluation process, with data sources represented by means of parchments.

Firstly, teachers attended a preliminary training session (Phase I) aimed at introducing the concept of a UoL and providing an overview of the main functionalities of Collage, which was employed by the initial designers at the MOSAIC course as well as by the teachers during evaluation so that they could analyse it. We chose Collage to support the evaluation process for a number of reasons. Firstly, Collage's user-friendly interface allowed teachers to easily analyse the UoL they had to reuse, even if they were not able to understand IMS LD computing formalism (XML language). In addition, Collage is a high-level IMS LD-compliant authoring tool that is specialized for CSCL (HernándezLeo et al., 2006), thus it may reduce the difficulties in modelling collaborative learning processes (Miao et al., 2005) but, at the same time, it maintains compliance with IMS LD Level A (IMS Global Learning Consortium, 2003). So teachers could evaluate the value and limitations of IMS LD UoLs without having specific XML writing skills. Finally, the same editor had been used by the original MOSAIC designers and therefore its use could be appropriate in this case too.

In the next phase, for simplicity's sake teachers were invited to analyse the UoL that comprised only the Jigsaw activity of the MOSAIC course (see Figure 1 and Section 2.1). In addition, they received a document illustrating the design scenarios in which they were about to participate (scenarios document). The document invited them to analyse the UoL by means of the Collage tool (Phase II) and then to instantiate it into three different sessions (Phase III), corresponding to three different realistic educational scenarios. The first scenario was characterized by the same contextual constraints of the MOSAIC session; in the second scenario the session involved more students (60) and more time available (six weeks) and the same communication tool (instant messaging tool); the third scenario dealt with 75 students and three weeks of available time, after which teachers were supposed to structure parallel sessions using a different communication tool (a forum). These scenarios were defined to stress particular design choices, especially related with good practices for the structuring of CSCL groups.

Teachers then had to document the main design elements characterizing the three sessions, which were the result of instantiating the same UoL into these three scenarios (thereby producing Design Annotation Document $I$ - DAD_I). Once completed, teachers were invited to analyse a Web-based tutorial which provided an SSR-based hypertextual and interactive modelling of the GRID Jigsaw UoL (Phase IV) (see Figure 4). Finally, teachers were asked to fill in another version of the Design Annotation Document (DAD_II) (Phase V) in which they annotated any change they wanted to apply to previous designed sessions once they had analysed information provided by the SSRs. 
These documents also allowed teachers to express their meta-reflections about the process in an open-text field.

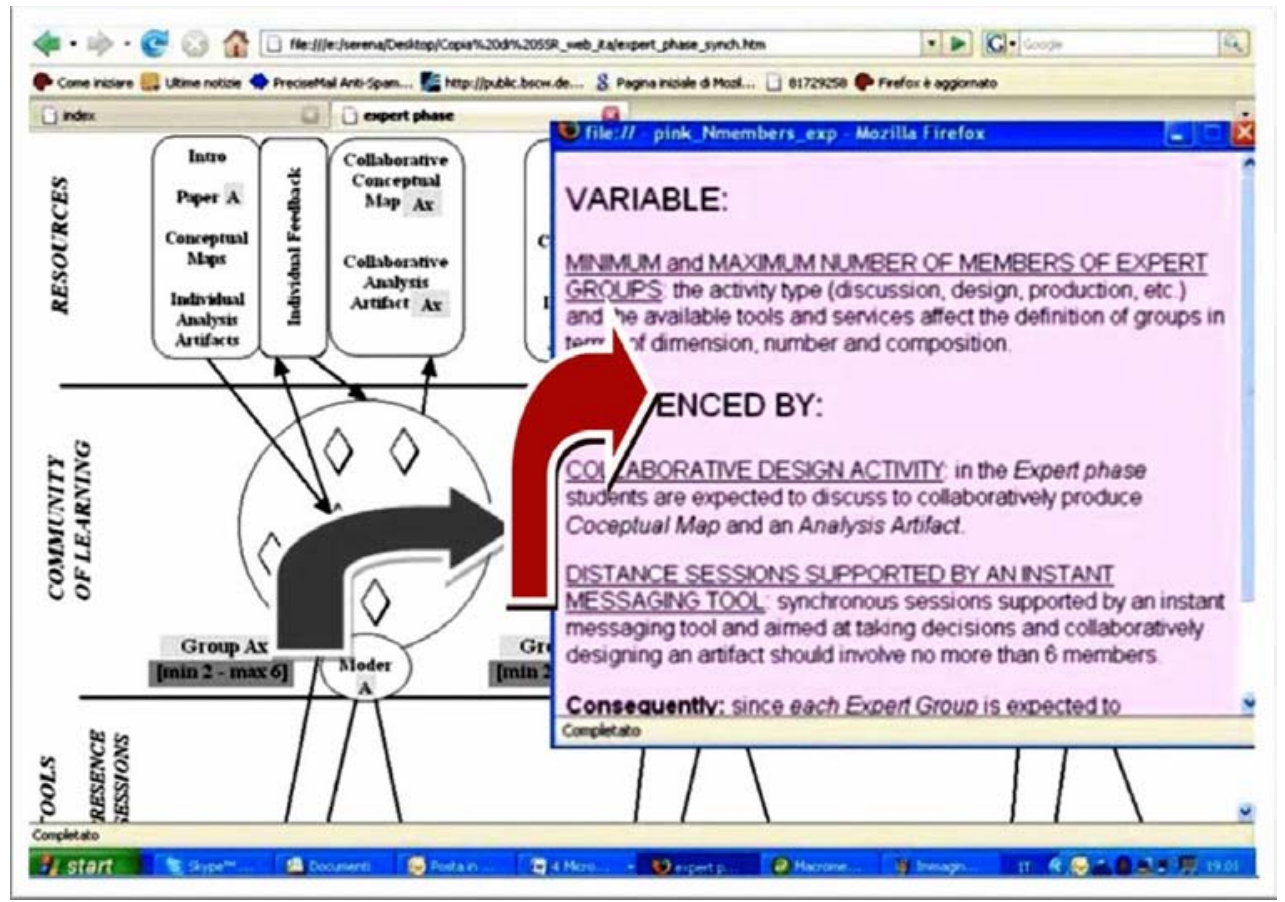

[Enlarge Image]

Figure 4. SSRs tutorial and pop-up mechanism for navigating variables and constraints.

This highly structured design process allowed the comparison of the different paths and choices taken by the teachers. Normally a design process is influenced by many variables, such as the characteristics of the teacher (experience, skills, preferences, attitudes), the educational context (contextual constraints, characteristics of the target learners, financial constraints), and the availability of resources and services (technologies, learning objects, learning designs) (Greeno et al., 1990). To foster comparability, we involved teachers with similar characteristics and experiences and we set a predefined design context characterized by fixed constraints. In particular, as mentioned before, the 10 teachers had similar characteristics in terms of their educational skills and experience with online learning design. In addition, their experience with UoLs and Collage had been made reasonably uniform by the initial training session. They also carried out their design process within the same realistic educational scenarios, which fixed some crucial context constraints (e.g., number of participants, academic context, technological constraints, timetable). Finally, the teachers could rely on the same resources (the $U o L$ ) and communication tools (an instant messaging tool in the first two scenarios and a forum in the third).

Within this design process we evaluated the teachers' choices on the basis of our initial issues of interest, aware also that in a multidimensional process such as this some of the variables were beyond our control.

A mixed evaluation method combining quantitative and qualitative data gathering was applied. Quantitative data are considered useful for showing trends. Qualitative results are used to confirm or reject those trends, to understand them, and to identify emergent features in the particular design situation. The same mixed evaluation was employed in 
previous case studies aimed at evaluating the functionalities and potentialities of Collage (Hernández-Leo et al., 2006).

Table $\underline{1}$ shows the different data sources employed in the evaluation process according to this mixed method. Teachers' characteristics were investigated with an initial selfadministered questionnaire (INITIAL_QUEST). The two Design Annotation Documents provided a form to describe the main design elements of the three sessions, firstly without the SSR support (DAD_I) and secondly with this kind of support (DAD_II). Finally, a questionnaire-based interview (FINAL-INTERVIEW) was carried out to collect teachers' opinions and feedback. Findings from the qualitative and quantitative data were triangulated to provide stronger evidence for drawing conclusions.

Table 1. Data sources for the evaluation process and labels used in the text to quote them.

Data source

Self-administered ex ante questionnaire

Design annotation documents

Final questionnairebased interview
Type of data

Quantitative and qualitative data about teachers' educational skills in learning design

Record of the main design elements characterizing the three sessions with respect to two different design situations (with and without SSR support)

Qualitative teachers' opinions and feedback
Labels

[INITIAL_QUEST]

[DAD_I]

[DAD_II]

[FINAL-

INTERVIEW]

\subsection{Results and discussion}

The initial questionnaire was aimed at evaluating whether the 10 teachers really had similar learning design skills and experiences. Data analysis suggests that (a) all of them had some or limited experience in structuring or managing online learning groups; (b) 8 out of 10 declared they knew some techniques or good practices to support CSCL and were able to list up two or three examples; (c) 8 out of 10 had heard about IMS LD UoLs, but only one teacher had ever seen a UoL and none had reused this kind of script. So the teachers' learning design skills were quite similar and even the initial training session provided them with a similar level of knowledge about IMS LD UoLs and Collage.

Data collected by means of the questionnaire-based final interview has been aggregated to identify trends and further used to select meaningful quotations from the open answers.

The Design Annotation Documents provided two types of qualitative data: the main design elements characterizing the three sessions and some meta-reflections on the process. Firstly, each designed session was evaluated according to a quality scale $(1=$ completely ineffective; 2 = partially ineffective; 3 = quite effective; 4 = completely effective). Note that during Phase III each participant designed three sessions and 
annotated them in [DAD_I]. Then during Phase V they could modify their previous designs, creating three new sessions, annotated in [DAD_II]. This evaluation was carried out by a researcher on the basis of three main criteria: (a) level of understanding of Jigsaw activity's flow and structure; (b) effectiveness of choices about group dimensions, taking into account the aim of groups and the tool supporting distance communication (see Section 2.2); (c) the creation of parallel sessions (or parallel courses) when the number of students is high, so as to set an ideal and effective number of participants in each session. These criteria refer to well-known CSCL heuristics and good practices (Kabanoff \& O'Brien, 1979; Trentin, 2001; Zang \& Ge, 2005) that are also valid for the SSR of the MOSAIC course. A further analysis classified improvements on the basis of the three aforementioned evaluation criteria, and finally basic quantitative processing (means, distribution) was performed on these data. These quantitative data were triangulated with the personal meta-reflections that some teachers annotated in the documents.

Table 2 summarizes the main findings and relates them to the three issues of interest of this evaluation process.

Table 2. Conclusions from the evaluation process.

\section{Evaluation goals}

A. When reusing UoLs, unskilled teachers lack information about how to effectively instantiate them, especially in terms of groups structuring.

B. SSRs provide complementary information to the ones provided by learning designs (UoLs).

\section{SSRs effectively support} unskilled teachers in reusing and instantiating scripts.

\section{Findings}

Support data

- [INITIAL_QUEST]

$\checkmark$ PHASE III: teachers encountered problems in the
instantiation process.

$\checkmark$ PHASE III: teachers lacked information about how to structure groups in the sessions.

PHASE V: teachers didn't lack information about how to structure groups in the sessions.

PHASE V: teachers perceived an effective support in designing sessions.

$\checkmark$ PHASE V: teachers added improvements on their designs concerning group structuring.

CONCLUSION: teachers evaluated positively SSRs.
- [DAD_I] and [DAD_II]: metareflections

- [FINALINTERVIEW].

- [FINALINTERVIEW].

- [DAD_I] and [DAD_II]: session rating

- [DAD_I] and [DAD_II]: metareflections

- [FINAL- 
Table 2. Conclusions from the evaluation process.

\title{
Evaluation goals
}

\author{
Findings
}

Support data

INTERVIEW].

The first two findings of Table $\underline{2}$ provide evidence that when reusing UoLs, unskilled teachers lack information about how to effectively instantiate them, especially in terms of group structuring. In particular, the quality level of the sessions described in Design Annotation Document I shows that teachers encountered problems in instantiating the predefined UoL according to the proposed scenarios. On a scale of 1-4 as mentioned above, the mean of quality level was 2.6 for the first (simpler) scenario, 2.2 for the second, and 2.1 for the third (the most complex). As to the first scenario, the one characterized by the same contextual constraints of the MOSAIC course, only four teachers created a completely effective session, each of which was based on the same group structuring implemented for the MOSAIC course. As to the second and the third scenarios, only one completely effective session was designed. Most teachers (6) misunderstood the nature of the Jigsaw activity, structuring groups without taking into account the main structural constraints (see Section 2.2) of this activity. This misunderstanding caused them to repeat the same mistake in both of the other sessions they designed in Phase III. In addition, while structuring groups they did not consider some heuristic constraints concerning group dimensions and assigned too many students to the same session. These data provide evidence that the teachers encountered some problems in instantiating the UoL. Further support comes from some of the comments provided by the teachers in the meta-reflection section of the Design Annotation Document 2. One teacher commented that 'constraints concerning groups dimensions and related to the selected communication tool were not explicit in the UoL.' While another one affirmed, 'By the analysis of the UoL, I couldn't understand that students should produce a collaborative artefact. I realized it when watching at SSRs.'

These assertions also support the second finding. During the first design process (Phase III) teachers lacked information about how to structure groups in the sessions. We can find analogous assertions analysing data from the final interview. According to 7 out of the 10 teachers, the information that Collage provided to formalize in a UoL was not sufficient to support them in the instantiation of an effective session. In addition, when asked more specifically about group structuring, 9 teachers out of the 10 re-stated that UoLs do not foster the expression of sufficient information about how to structure groups in terms of number, dimension, and composition. This is not a limitation inherent to the specific tool that was employed, but in the IMS LD language to which Collage is compliant. In addition, the concrete UoL provided as much information as it could within the IMS LD specification. The teachers acknowledged that it was well described, but they perceived that UoLs in general do not provide sufficient modes of expression to support the instantiation process. Some of them confirmed that the UoL 'lacked precise information about how to structure groups' and 'the information provided is sufficient only for expert designers' but 'is not sufficient for inexperienced teachers.' Others affirmed this saying, 'design steps are well structured but the UoL lacks detailed information about how to structure groups'; 'these pieces of information are not provided unless the designer puts them in the open-text fields devoted to descriptions; what is provided is organizational information.' 
The third and fourth findings in Table 2 suggest that SSRs provided complementary information to what is offered through learning designs (UoLs). In particular, qualitative and quantitative data from the final interview show that in the second design process (Phase V in which support was provided by the SSRs):

- teachers did not lack information about how to structure groups in the sessions;

- teachers perceived an effective support in designing sessions deriving partly from UoL description and partly from the SSRs.

According to 7 out of the 10 teachers, information provided by the joint use of a UoL and the corresponding SSR was sufficient to instantiate an effective session. In addition, 9 out of the 10 teachers affirmed that this joint use provided sufficient information about how to effectively instantiate groups (in terms of number, dimension, and composition) in a session. These findings are also supported by some teachers' comments, for example: 'they are complementary because they provide two different points of view on the same process'; 'they complete each other, providing fundamental information about how to instantiate these scripts'; 'they are complementary because UoLs provide support for general-level choices, while SSRs provide support for the more detailed group-structuring design step.' As to SSRs, teachers also commented that 'information about how to structure groups is contained in SSRs'; 'this information is rendered explicit by SSRs by means of a number of variables represented with different colours'; 'the groups structuring results from the analysis of the variables that SSRs underline.'

Finally the last two findings shown in Table $2 \underline{2}$ support the argument that SSRs effectively supported unskilled teachers in reusing and instantiating scripts. In particular, comparison of the Design Annotated Documents confirms that in the second design process (Phase V, with the support of SSRs), teachers improved the structuring of groups. The mean quality level changed from 2.6 to 3.1 in the first scenario, from 2.2 to 3.2 in the second, and from 2.1 to 3.2 in the third. In particular, 8 out of the 10 teachers improved their designs somehow; 3 of them added improvements in all scenarios, 3 in two scenarios, and 2 teachers in only one scenario. Classifying the improvements according to the aforementioned evaluation criteria we can find that:

- $\quad 31 \%$ of them concerned the level of understanding of Jigsaw activity's flow and structure;

- $\quad 41 \%$ concerned the effectiveness of choices about group dimensions, taking into account the aim of groups and the tool supporting distance communication;

- the remaining $28 \%$ concerned the effective use of parallel sessions to set an ideal number of participants in each session.

Additionally in the meta-reflection section of Design Annotated Document II teachers' comments included 'SSR has been useful to immediately understand that I was wrong about groups structuring'; and 'SSRs allow revisiting the UoL and understanding if the design has gone far afield.'

Quantitative and qualitative data from the final interview provided support for the last finding: that teachers evaluated SSRs positively. According to 8 of the 10 teachers, SSRs provided a very useful support to instantiate a UoL. Qualitative comments from the final interview reinforce this: 'SSR helps to give an overall vision of the process' and 
'it provides support in the more detailed and specific steps of the design process.' Others affirmed that 'it provides a global and complete vision of the process'; 'it provides information that are not embedded in the UoL such as information about groups or the moderators'; and 'without SSR you're likely to do mistakes in the design process.' Only two teachers affirmed that SSRs were 'quite useful,' both arguing that their utility depends on the level of ID competencies of a teacher. In addition, 8 of the 10 teachers declared that SSRs are quite easily understood. As two teachers mentioned: 'constraints are explicit and clear so SSRs don't leave space for misunderstandings or wrong interpretations'; and 'at the first sight information I needed came up.' Only two teachers declared that SSR were 'a little complex to be understood,' commenting 'it is not so immediate but, once understood, the logic [by interacting with the system] provides very precious information'; 'the language is a bit specific but on the whole it is very complete'; and 'there is much information that requires time to be analyzed, but it is very useful if provided together the UoL.' Some teachers provided suggestions such as 'it lacks a preview or a simulation of the session' or 'it would be nice if a designer could manipulate diagrams to build a personalized activity structure.'

Based on the above mixed evaluation process in a controlled environment and using realistic scenarios with practitioners relatively unskilled with respect to the learning design, there is significant triangulated evidence that supports the aforementioned findings. Thus, some light has been shed on the issues of interest in this study, upholding our initial assumption: SSRs play a complementary role to learning designs (UoLs) by formulating shared representations of group structures and support unskilled teachers in reusing and instantiating scripts for their particular educational context.

\section{Conclusions}

This article has illustrated some challenges that a teacher can encounter when reusing a CSCL script and proposed SSRs as a possible solution. Supporting the representation of groups' social structures, as well as the structural and heuristic constraints embedded in the script, SSRs effectively support the script instantiation into different educational contexts. In particular, SSRs can overcome some well-known limitations of IMS LD concerning the representation of groups, allowing their visualization as a specific entity, characterized by social structures (number, dimension, composition) and explicit relations with the whole community of learning (e.g., individuals, teachers, experts, moderators). A collaborative activity is no longer described exclusively according to the activity flow and the associated resources, but on the basis of the structures which characterize each task or phase. This perspective provides complementary information to a teacher who may analyse a UoL in order to reuse and instantiate it in his/her own context.

Findings from the mixed method evaluation which involved teachers in a controlled design experience based on realistic scenarios clearly point out the support provided by SSRs, both in terms of the improvements made to designs and the positive perceptions of participants. This shows that SSRs can support the representation of a learning design through a complementary focus on the social structures' perspective, and as such can be effectively associated with a script to support its instantiation.

One should bear in mind that SSRs represent a part of the context in any learning situation, even when individuals learn within a social group (class or community), 
although its importance is greater in the case of CSCL due to its inherent complexity and the importance of social structures in collaborative learning. Also, the main contribution was illustrated through the main standard learning design language, although it may be applied to other computer interpretable or visual languages that have been proposed in the literature.

As to future work, in the short term a more rigorous formalization of SSR semantics will be carried out. Additionally, there is a clear need to study the possibility of sharing SSRs together with the associated script, so as to allow a teacher to effectively select a script for reuse on the basis of the embedded constraints and their coherence with the specific contextual situation. At the software support level, Collage may be modified so as to allow the creation of SSRs while formalizing the UoL, in such a way that both elements can be shared together to support a possible reuse and the subsequent instantiation process. Finally, a new Collage version may be developed to dynamically show during the script authoring all the implications on constraints represented in the SSRs, thus supporting a more aware authoring process.

\section{Acknowledgements}

This work of the GSIC/EMIC group at the University of Valladolid, Spain, has been partially funded by projects IST-FP6-034567, TSI2005-08225-C07-04, and VA009A05. Special thanks are due to the 10 teachers participating in the SSRs' validation process. A special mention is due to Luigi Sarti and Paola Forcheri (Italian National Research Council) for their precious feedback.

\section{Notes on contributors}

Serena Alvino is consulting professor for the E-learning for adults and organizations course at the University of Genoa. Since 2002, she has been collaborating with the Institute for Educational Technologies of the Italian National Research Council investigating the use of CSCL, focusing on the instructional design of collaborative online courses.

Juan I. Asensio-Pérez is an associate professor at the University of Valladolid, Spain. His research interests include distributed systems particularly, distributed CSCL applications, and integrated systems and network management.

Yannis A. Dimitriadis is a professor at the University of Valladolid, Spain. His research interests include CSCL and distributed systems.

Davinia Hernández-Leo lectures in the Department of Information and Communications Technologies of Pompeu Fabra University and is a member of the GTI research group. Davinia's main research interests are educational telematics, CSCL, techniques for the design of educational situations, and learning technology standards and specifications.

\section{References}


- 1. Alvino, S. (2008) Computer Supported Collaborative Learning e riusabilità: Un approccio all'integrazione di risorse riusabili in processi di apprendimento collaborativo [CSCL and reusability: An approach to the integration of reusable resources in collaborati University of Genoa , Italy — Unpublished doctoral thesis [your library's links]

- 2. Botturi, L. and Stubbs, T. (eds) (2007) Handbook of visual languages for instructional design: Theories and practices Information Science Reference , Hershey, PA [your library's links]

- 3. de la Fuente, L., Pardo, A., Delgado, C., Asensio, J. I. and Dimitriadis, Y. A. Diaz, P., Kinshuk, I. Aedo and Mora, E. (eds) (2008) Collaborative learning models on distance scenarios: A case study. Proceedings of the 8th IEEE International Conference on Advanced Learning Technologies Los Alamitos, CA IEEE Computer Society Press pp. 278-282. [your library's links]

- 4. Dillenbourg, P. Kirschner, P. A. (ed) (2002) Over-scripting CSCL: The risks of blending collaborative learning with instructional design. Three worlds of CSCL: Can we support CSCL? pp. 61-91. Open Universiteit Nederland , Heerlen, the Netherlands [your library's links]

- 5. Dillenbourg, P. and Tchounikine, P. (2007) Flexibility in macro-scripts for computer-supported collaborative learning. Journal of Computer Assisted Learning 23:(1) , pp. 1-7. [your library's links] [ crossref ]

- 6. Escobedo, J. P., de la Fuente, L., Gutiérrez, L., Pardo, A. and Delgado, C. (2007) Implementation of a learning design run-time environment for the.LRN learning management system. Adaptation and IMS learning design [Special issue]. Journal of Interactive Media in Education - Retrieved March 29, 2009, from http://www-jime.open.ac.uk/2007/07/ [your library's links]

- 7. Greeno, J. G., Korpi, M. K., Jackson, D. N. and Michalchik, V. S. (1990) Processes and knowledge in designing instruction Stanford University, Palo Alto, CA [your library's links]

- 8. Hernández-Gonzalo, J. A., Villasclaras-Fernández, E. D., Hernández-Leo, D., Asensio-Pérez, J. I. and Dimitriadis, Y. A. Diaz, P., Kinshuk, I. Aedo and Mora, E. (eds) (2008) InstanceCollage: A graphical tool for the particularization of role/group structures in pattern-based IMS-LD collaborative scripts.

Proceedings of the 8th IEEE International Conference on Advanced Learning Technologies Los Alamitos, CA IEEE Computer Society Press pp. 506-510. [your library's links]

- 9. Hernández-Leo, D., Asensio-Pérez, J. I. and Dimitriadis, Y. (2005) Computational representation of collaborative learning flow patterns using IMS learning design. Educational Technology \& Society 8:(4) , pp. 75-89. [your library's links]

- 10. Hernández-Leo, D., Harrer, A., Dodero, J. M., Asensio-Pérez, J. I. and Burgos, D. (2007) A framework for the conceptualization of approaches to 'Create-by-Reuse' of learning design solutions. Journal of Universal Computer Science 13:(7) , pp. 991-1001. [your library's links]

- 11. Hernández-Leo, D., Villasclaras-Fernández, E. D., Asensio-Pérez, J. I. and Dimitriadis, Y. Botturi, L. and Stubbs, T. (eds) (2007) Diagrams of learning flow patterns' solutions as visual representations of refinable IMS learning design templates. Handbook of visual languages for instructional design: Theories and practices pp. 394-412. Information Science Reference , Hershey, PA [your library's links] 
- 12. Hernández-Leo, D., Villasclaras-Fernández, E. D., Asensio-Pérez, J. I., Dimitriadis, Y., Jorrín-Abellán, I. M. Ruiz-Requies, I. et al. (2006) COLLAGE: A collaborative learning design editor based on patterns. Educational Technology \& Society 9:(1) , pp. 58-71. [your library's links]

- 13. IMS Global Learning Consortium (2003) IMS learning design version 1.0 final specification - Retrieved October 15, 2008, from http://www.imsglobal.org/learningdesign/index.cfm [your library's links]

- 14. Kabanoff, B. and O'Brien, G. E. (1979) The effects of task type and cooperation upon group products and performance. Organizational Behaviour and Human Performance 23:(2) , pp. 63-181. [your library's links]

- 15. Kaleidoscope (2003-2009) - Retrieved February 28, 2009, from http://www.noe-kaleidoscope.org/pub/

- 16. Koper, R. (2006) Current research in learning design. Educational Technology \& Society 9:(1) , pp. 13-22. — Retrieved October 9, 2008, from http://www.ifets.info/journals/9_1/3.pdf [your library's links]

- 17. Koschmann, T. D. (1996) CSCL: Theory and practice of an emerging paradigm Lawrence Erlbaum Associates, Mahwah, NJ [your library's links]

- 18. .LRN - Retrieved March 29, 2009, from http://www.dotlrn.org/

- 19. Miao, Y., Hoeksema, K., Hoppe, H. U. and Harrer, A. Koschmann, T., Suthers, D. and Chan, T. W. (eds) (2005) CSCL scripts: Modelling features and potential use. Proceedings of the Computer Supported Collaborative Learning 2005: The next 10 years! pp. 423-432. Lawrence Erlbaum Associates , Mahwah, NJ [your library's links]

- 20. Open University Cloudworks - Retrieved October 25, 2008, from http://cloudworks.open.ac.uk/

- 21. Paquette, G., Léonard, M., Ludgren-Cayrol, K., Mihaila, S. and Gareau, D. (2005) Learning design based on graphical modelling. Educational Technology \& Society 9:(1) , pp. 97-112. — Retrieved March 29, 2009, from http://ares.licef.teluq.uqam.ca/Portals/29/docs/pub/ingenierie/Graphic\%20Know ledge-based\%20V2.pdf [your library's links]

- 22. Persico, D. and Sarti, L. Chiazzese, G., Allegra, M., Chifari, A. and Ottaviano, S. (eds) (2005) Social structures for online learning: A design perspective. Proceedings of Conference on Methods and Technologies for Learning pp. 51-59. Wit Press , Palermo, Italy [your library's links]

- 23. Silber, K. H. (2007) A principle-based model of instructional design: A new way of thinking about and teaching ID. Educational Technology 47:(5) , pp. 3437. [your library's links]

- 24. Stake, R. E. (1995) The art of case study research Sage, London [your library's links]

- 25. TENCompetence ReCourse learning design editor - Retrieved March 29, 2009, from http://www.tencompetence.org/ldauthor

- 26. Trentin, G. Maddux, C. D. and Johnson, L. (eds) (2001) Designing online courses. The Web in higher education: Assessing the impact and fulfilling the potential pp. 47-66. Haworth Press , London [your library's links]

- 27. Vignollet, L., David, J. -P., Ferraris, C., Martel, C. and Lejeune, A. Kommers, P., Kirschner, P., Sampson, D., Kinshuk and Koper, R. (eds) (2006) Comparing educational modeling languages on a case study. ICALT 2006: Advanced technologies for life-long learning. Proceedings of the 6th IEEE International Conference on Advanced Learning Technologies Los Alamitos, CA IEEE Computer Society Press pp. 1149-1151. [your library's links] 
- 28. Weinberger, A., Collar, I., Dimitriadis, Y., Mäkitalo-Siegl, K. and Fischer, F. Balacheff, N., Ludvigsen, S., de Jong, T., Lazonder, A., Barnes, S. and Montandon, L. (eds) (2008) Computer-supported collaboration scripts:

Perspectives from educational psychology and computer science. Technologyenhanced learning: Principles and products pp. 155-173. Springer-Verlag , Dordrecht, the Netherlands [your library's links]

- 29. Zang, K. and Ge, X. Figueiredo, A. D. and Afonso, A. P. (eds) (2005)

Dynamics of online collaboration: Team task, team development, peer relationship and communication media. Managing learning in virtual settings: The role of context pp. 98-116. Information Science Publishing, London [your library's links]

\section{List of Figures}

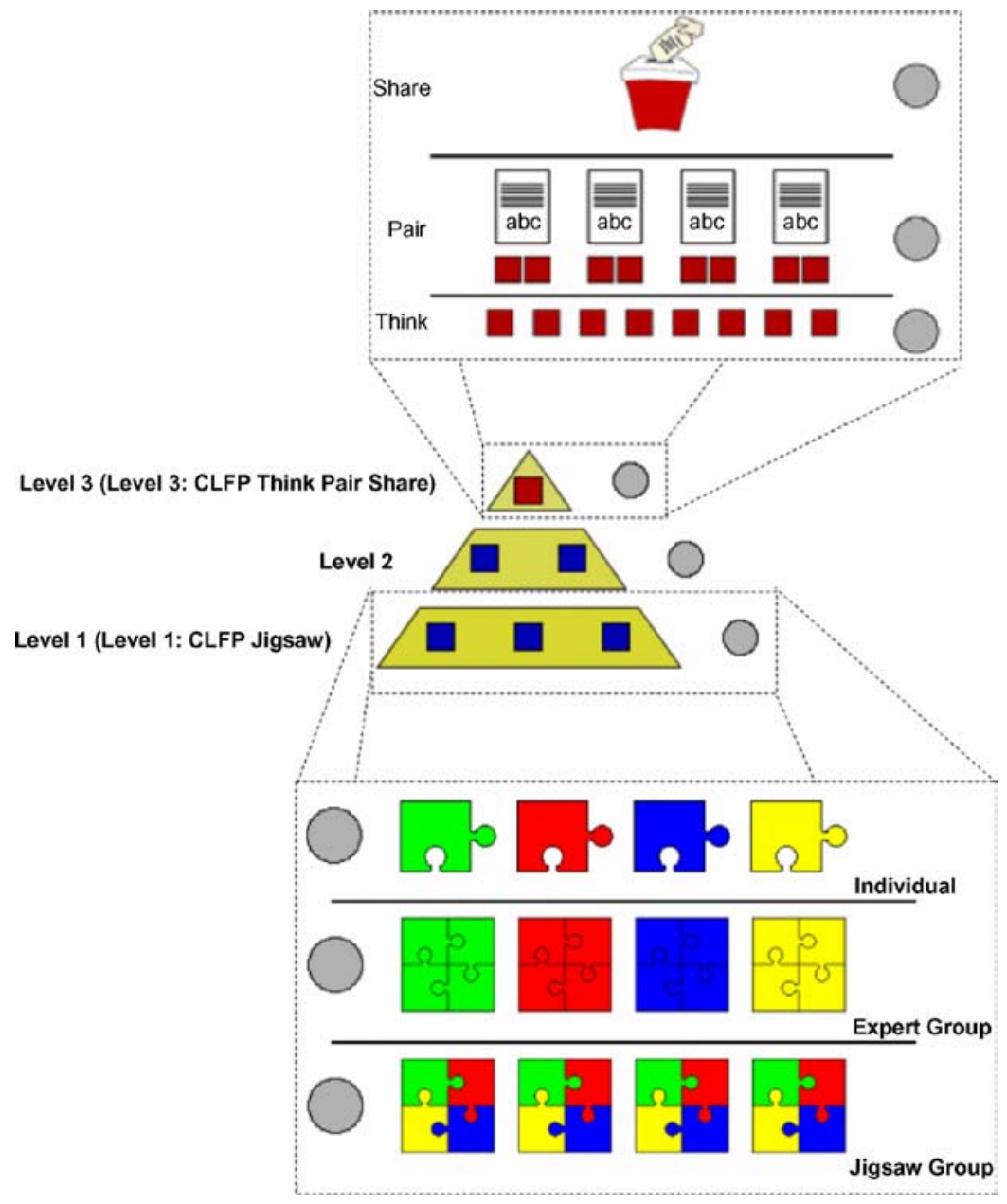

[Enlarge Image] 
Figure 1. CSCL script of the MOSAIC course, as represented in the Collage tool.

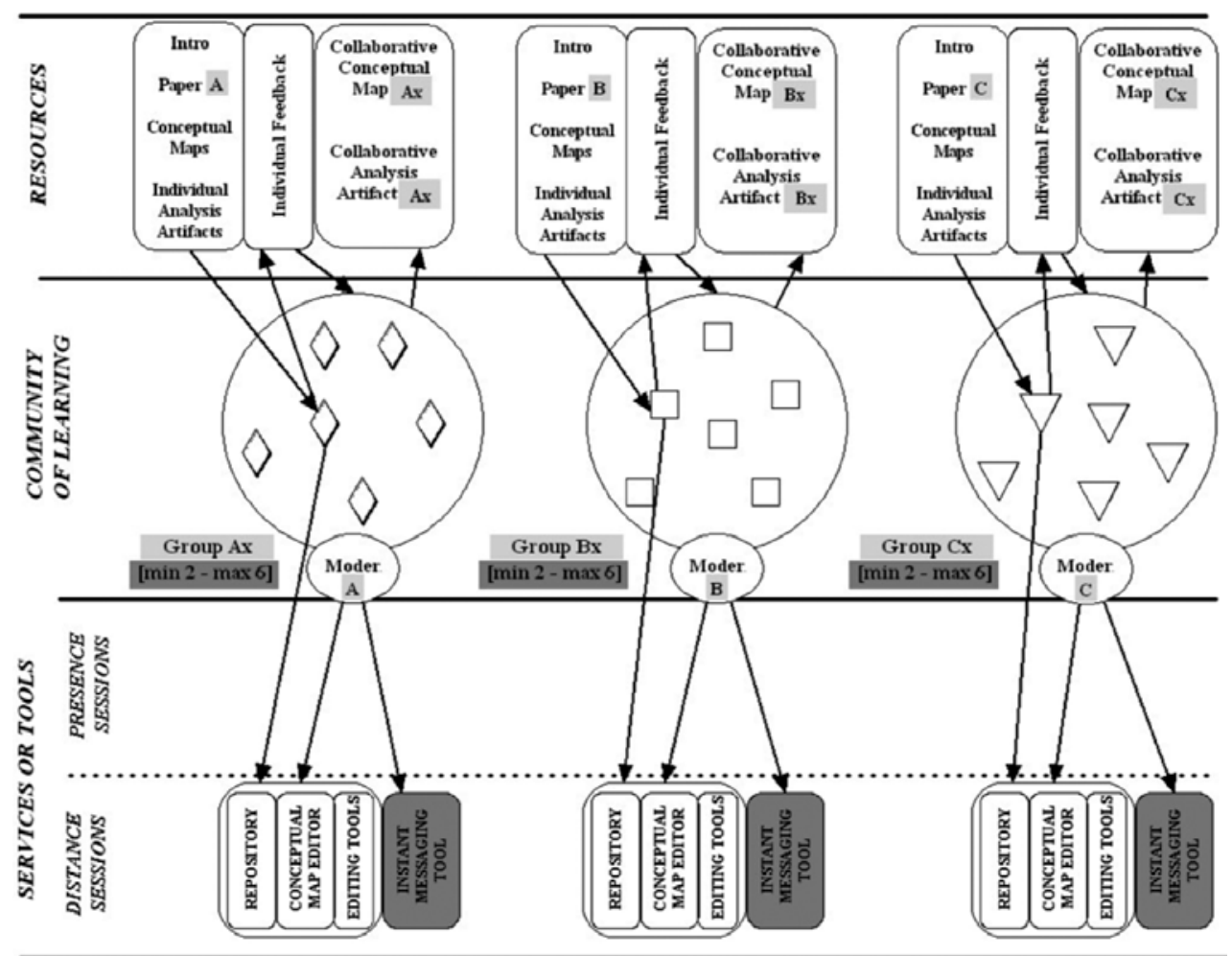

[Enlarge Image]

Figure 2. Instance level SSR of the MOSAIC Jigsaw's expert groups phase.

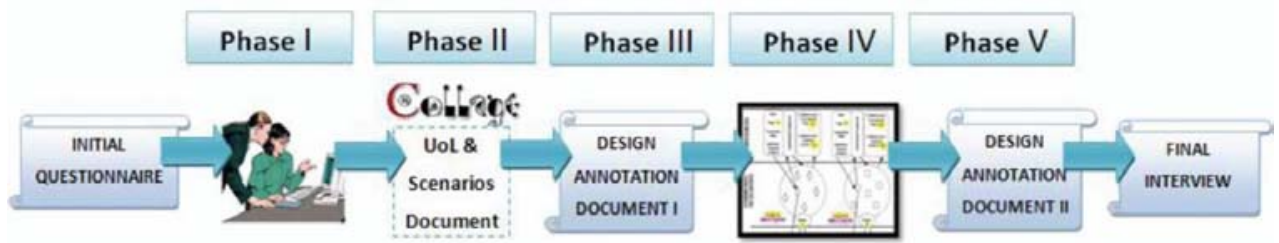

[Enlarge Image]

Figure 3. Overview of the evaluation process, with data sources represented by means of parchments. 


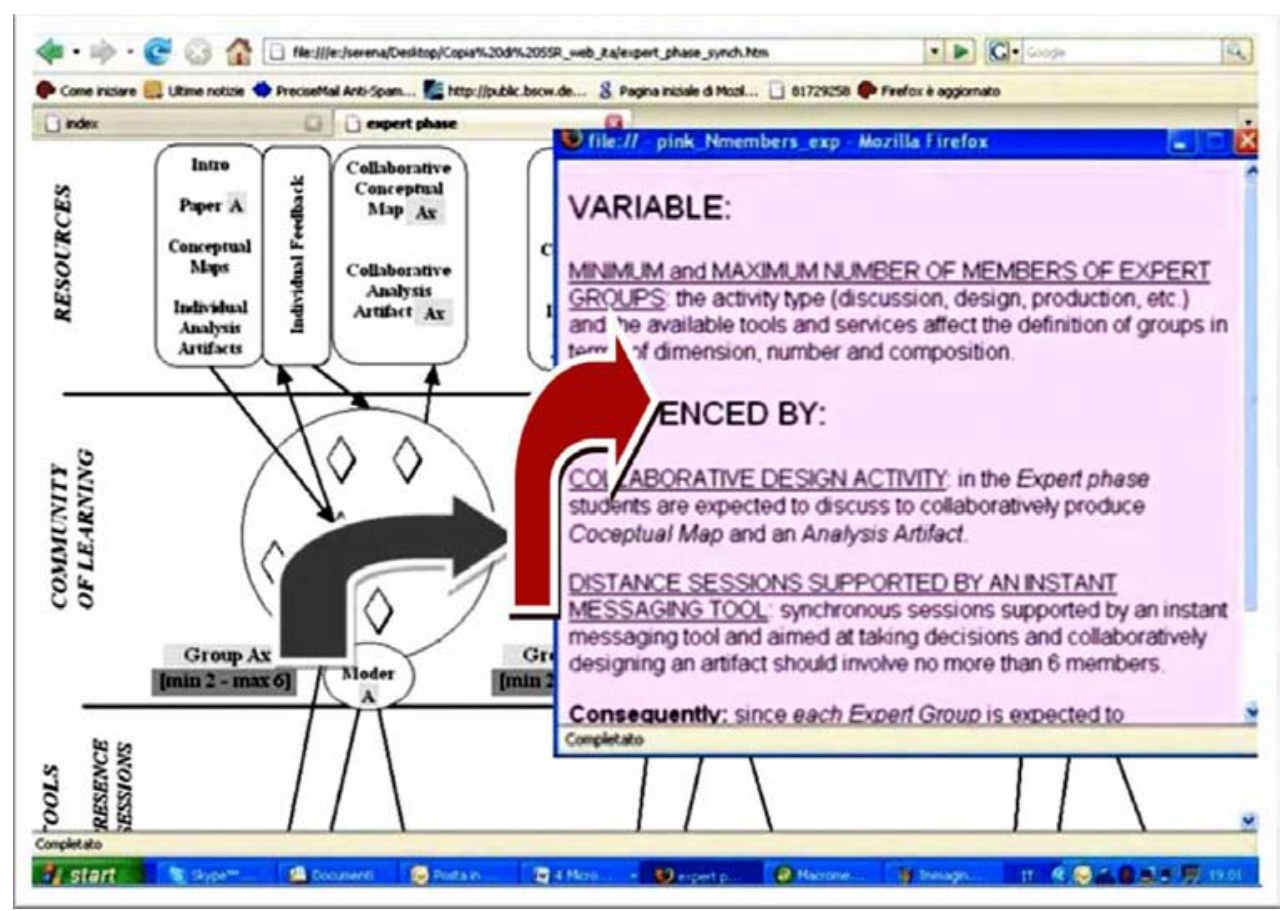

[Enlarge Image]

Figure 4. SSRs tutorial and pop-up mechanism for navigating variables and constraints.

\section{List of Tables}

Table 1. Data sources for the evaluation process and labels used in the text to quote them.

\section{$\begin{array}{lll}\text { Data source } & \text { Type of data } & \text { Labels }\end{array}$}

Self-administered

ex ante

questionnaire

Quantitative and qualitative data about teachers' educational skills in learning design [INITIAL_QUEST]

Record of the main design elements

Design annotation characterizing the three sessions with respect documents to two different design situations (with and without SSR support)

Final questionnairebased interview

Qualitative teachers' opinions and feedback

[DAD_I]

[DAD_II]

[FINAL-

INTERVIEW]

Table 2. Conclusions from the evaluation process.

Evaluation goals

A. When reusing UoLs, unskilled teachers lack information about
Findings

PHASE III: teachers
Support data

- [INITIAL_QUEST] 
Table 2. Conclusions from the evaluation process.

\section{Evaluation goals}

Findings

Support data

how to effectively instantiate encountered problems in the - [DAD_I]: session them, especially in terms of groups instantiation process. rating structuring.

B. SSRs provide complementary information to the ones provided by learning designs (UoLs).

C. SSRs effectively support unskilled teachers in reusing and instantiating scripts.

$\checkmark$ PHASE III: teachers
lacked information about
how to structure groups in
the sessions.

- [DAD_I] and [DAD_II]: metareflections

- [FINALINTERVIEW].

$\checkmark$ PHASE V: teachers didn't lack information about how to structure groups in the sessions.

$\checkmark$ PHASE V: teachers

- [FINALINTERVIEW]. perceived an effective support in designing sessions.

$\checkmark$ PHASE V: teachers added improvements on their designs concerning group structuring.

$\checkmark$ CONCLUSION:

teachers evaluated positively SSRs.
- [DAD_I] and [DAD_II]: session rating

- [DAD_I] and [DAD_II]: metareflections

- [FINALINTERVIEW]. 